\title{
Adaptive Fault-Tolerant $H \infty$ Power and Rate Control for Wireless Networks with Receiver Failures
}

\author{
Cunwu Han, Xiaofei Li, Dehui Sun, and Zhijun Li
}

\begin{abstract}
This paper investigates adaptive fault-tolerant $H \infty$ power and rate control for wireless networks. Based on the new joint power and rate control model, an indirect adaptive fault-tolerant controller via dynamic output feedback is presented for receiver fault compensations. The adaptive $H \infty$ performance of the closed-loop system is analyzed based on the linear matrix inequality (LMI). Simulation results are given to demonstrate the effectiveness of the proposed controller.
\end{abstract}

Index Terms-Power and rate control, wireless networks, fault-tolerant control, $H \propto$ control.

\section{INTRODUCTION}

Power and rate control are important requirements for wireless networks. On one hand, the power transmitted by individual nodes must be high enough to ensure a reliable connection, while this may cause interference to other nodes in the network. Therefore, efficient power control is an essential requirement for wireless networks. On the other hand, the transmission data rates need to be high enough in order to efficiently support a large variety of services such as voice, video, data and/or their mixture, while high date rates may cause date bursts in the networks. This needs effective rate control. Additionally, the transmission power and data rate will be affected each other, this needs power and rate control to be jointly considered.

Joint power and rate control problem has received much attention during recent decades, and many effective algorithms have been presented for code-division multiple access (CDMA) wireless communication networks [1-6] and for wireless ad hoc networks [7-9]. However, all the proposed algorithms are under a full reliability assumption that all control components of the systems are in good working conditions. Unfortunately, faults of control components often occur in actual environments of wireless networks, and this may lead to unsatisfactory performance of the closed-loop system or even instability. To the best knowledge of the authors, there is only a few results on fault-tolerant power and rate control for wireless networks with control component faults reported in the literature [10,11].

How to compensate for the component faults is a challenging issue for control systems. Fault-tolerant control (FTC), as one of typical system design methods with control

Manuscript received April 27, 2012; revised June 8, 2012. This work was supported in part by the National Natural Science Foundation of China under Grant 61174116, Beijing high-level talents project, and Research Fund of North China University of Technology.

The authors are with the Beijing Key Laboratory of Fieldbus Technology and Automation, North China University of Technology, Beijing 100144 China (e-mails: cwhan, sundehui, 1zj78)@ncut.edu.cn, gmlxf123@ yahoo.cn). component faults, has received much attention during recent decades, which keeps the system safe to achieve satisfactory performances whenever components are healthy or faulty. The existing fault-tolerant controllers can be divided into two design approaches, i.e., passive approach and active approach. Robust control is useful method in passive approach, in which linear matrix inequality (LMI) methods can be used to describe the performances of fixed gain closed-loop systems [12-17]. While adaptive control is very important technique in active approach, in which the parameters can be adjusted online to ensure reliability of closed-loop systems in the presence of a wide range of unknown faults [18-23].

In previous work, we presented adaptive fault-tolerant $H_{\infty}$ power and rate controller for wireless networks with transmitter faults via state feedback [10] and via dynamic output feedback [11], respectively. This paper considers receiver faults, extends the results proposed in [22] for linear continuous-time systems with sensor failures to discrete-time systems and presents an adaptive fault-tolerant $H_{\infty}$ power and rate controller for wireless networks with receiver faults at the master node. The adaptive $H_{\infty}$ performance is analyzed based on linear matrix inequalities (LMIs). Simulation results are given to demonstrate the effectiveness of the proposed algorithm.

The rest of this paper is organized as follows: The power and rate control are described in Section II. An adaptive fault-tolerant $H_{\infty}$ power and rate controller is designed and the performance is analyzed in Section III. Simulation results are given in Section IV. And Section V concludes the paper.

\section{POWER AND RATE CONTROL}

In this section, we first briefly describe the power and rate control in Subsection $A$ and $B$, respectively; and then derive the joint power and rate control for wireless networks without and with receiver faults in Subsection $C$ and $D$, respectively; finally give the problem formulation in Subsection $E$.

\section{A. Power Control}

Consider the uplink channel of wireless networks, in which $n$ nodes share the same channel. For any $i$-th node, the received signal-to-interference ratios (SIR) at the master node can be expressed as

$$
\gamma_{i}(k)=\frac{G_{i i}(k) p_{i}(k)}{\sum_{\substack{j=1 \\ j \neq i}}^{n} G_{i j}(k) p_{j}(k)+\sigma_{i}^{2}},
$$


where $G_{i j}$ is the link gain between the $j$ th node and the master node of the $i$ th cell, $p_{j}(k)$ is the transmit power of the $j$ th node, $\sigma_{i}^{2}$ is the receiver noise power. $[1-3]$

The power control algorithm (in $\mathrm{dB}$ scale) is chosen as

$$
\bar{p}_{i}(k+1)=\bar{p}_{i}(k)+\alpha_{i}\left[\bar{\gamma}_{i}^{*}(k)-\bar{\gamma}_{i}(k)\right],
$$

where $\alpha_{i}$ is a step-size factor, $\gamma_{i}^{*}(k)$ and $\gamma_{i}(k)$ are the desired and actual SIRs, respectively; $\bar{p}_{i}(k)=10 \log p_{i}(k)$, $\bar{\gamma}_{i}^{*}(k)=10 \log \gamma_{i}^{*}(k)$, and $\bar{\gamma}_{i}(k)=10 \log \gamma_{i}(k)$.

Now let

$$
\beta_{i}(k)=\frac{G_{i i}(k)}{\sum_{\substack{j=1 \\ j \neq i}}^{n} G_{i j}(k) p_{j}(k)+\sigma_{i}^{2}},
$$

then (1) can be rewritten as

$$
\gamma_{i}(k)=\beta_{i}(k) p_{i}(k),
$$

or, in $\mathrm{dB}$ scale,

$$
\bar{\gamma}_{i}(k)=\bar{\beta}_{i}(k)+\bar{p}_{i}(k),
$$

where $\bar{\beta}_{i}(k)$ is referred to as the effective link gain. Now introduce the random walk model in [1-3] for $\bar{\beta}_{i}(k)$ as

$$
\bar{\beta}_{i}(k+1)=\bar{\beta}_{i}(k)+n_{i}(k),
$$

where $n_{i}(k)$ is a white Gaussian noise with variance $\sigma_{n}^{2}$. It is clear from (2), (5) and (6) that the achieved $\bar{\gamma}_{i}(k)$ can be written as

$$
\bar{\gamma}_{i}(k+1)=\left(1-\alpha_{i}\right) \bar{\gamma}_{i}(k)+\alpha_{i} \bar{\gamma}_{i}^{*}(k)+n_{i}(k) .
$$

\section{B. Rate Control}

In this paper, we choose the transmission data rate, $f_{i}(k)$, according to the following algorithm as in $[1-3,11]$

$$
f_{i}(k+1)=f_{i}(k)+\mu\left[d(k)-c_{1}(k) f_{i}(k)\right],
$$

where $\mu>0$ is a step-size factor, $d(k)$ is the increase rate per iteration, which is a white Gaussian noise with variance $\sigma_{d}^{2}$, and $c_{1}(k)$ is the amount of congestion. Equation (8) is a typical rate control strategy incorporated in wireless networks and in computer networks.

The actual SIR, according to the capacity formula of Shannon, should be chosen no less than the desired $\gamma_{i}^{*}(k)$ in order to achieve the data rate $f_{i}(k)$ given by

$$
f_{i}(k)=\frac{1}{2} \log _{2}\left[1+\gamma_{i}^{*}(k)\right]
$$

and $\gamma_{i}^{*}(k) \gg>1$. Then, the desired SIR $\gamma_{i}^{*}(k)$ can be written as

$$
\bar{\gamma}_{i}^{*}(k+1)=\left[1-\mu c_{1}(k)\right] \bar{\gamma}_{i}^{*}(k)+\bar{\mu} d(k),
$$

\section{Joint Power and Rate Control without Receiver Faults}

For simplicity of notation, in the following, the node index $i$ is dropped. And we introduce the two dimensional state vector

$$
x(k)=\left[\begin{array}{c}
\bar{\gamma}(k) \\
\bar{\gamma}^{*}(k)
\end{array}\right],
$$

then from (7) and (10), we can obtain

$$
x(k+1)=\left[\begin{array}{cc}
1-\alpha & \alpha \\
0 & 1-\mu c_{1}(k)
\end{array}\right] x(k)+\left[\begin{array}{cc}
1 & 0 \\
0 & \bar{\mu}
\end{array}\right]\left[\begin{array}{l}
n(k) \\
d(k)
\end{array}\right],
$$

or, more compactly,

$$
x(k+1)=A x(k)+B_{1} w(k)
$$

where

$$
A=\left[\begin{array}{cc}
1-\alpha & \alpha \\
0 & 1-\mu c_{1}
\end{array}\right], \quad B_{1}=\left[\begin{array}{cc}
1 & 0 \\
0 & \bar{\mu}
\end{array}\right], \quad w(k)=\left[\begin{array}{c}
n(k) \\
d(k)
\end{array}\right],
$$

where $w(k)$ is a zero-mean random vector with covariance matrix

$$
Q=E\left\{w(k) w^{T}(k)\right\}=\left[\begin{array}{cc}
\sigma_{n}^{2} & 0 \\
0 & \sigma_{d}^{2}
\end{array}\right] \leq \rho_{w} I .
$$

In order to drive the actual SIR $\gamma_{i}(k)$ towards the desired value of SIR $\gamma_{i}^{*}(k)$, we employ a control sequence $u(k)$ to be determined in (13) as follows

$$
x(k+1)=A x(k)+B u(k)+B_{1} w(k) .
$$

Remark 1: We can choose some given $2 \times 2$ matrix $B$ and $2 \times 1$ control sequence $u(k)$ in (16), for example, let

$$
u(k)=\left[\begin{array}{l}
u_{p}(k) \\
u_{f}(k)
\end{array}\right]
$$

denote the individual entries of $u(k)$ to be designed. Then the inclusion of the term $B u(k)$ in (16) amounts to adding a power control signal $u_{p}(k)$ to the power update (2), and a rate control signal $u_{f}(k)$ to the desired SIR update (10).

Suppose for generality that we have access to output measurements that are related to the state vector as follows

$$
y(k)=C x(k)+D w(k),
$$

for some known matrices $C$ and $D$.

Remark 2: Usually, we can let $C=I$ so that the entries of $y(k)$ correspond to noisy measurements of the actual and desired SIR levels, $\left\{\bar{\gamma}(k), \bar{\gamma}^{*}(k)\right\}$. The matrix $D$ can be chosen according to the measurement noise.

The objective of joint power and rate control for wireless networks is to drive the received $\operatorname{SIR} \gamma_{i}(k)$ at the master node towards the desired SIR $\gamma_{i}^{*}(k)$ efficiently. To this end, we choose the controlled output $z(k)=C_{1} x(k)$ and $C_{1}=\left[\begin{array}{ll}1 & -1\end{array}\right]$. This choice of $C_{1}$ yields

$$
z(k)=\bar{\gamma}(k)-\bar{\gamma}^{*}(k),
$$


so that $z(k)$ is a measure of the difference between $\bar{\gamma}(k)$ and $\bar{\gamma}^{*}(k)$.

\section{Joint Power and Rate Control with Receiver Faults}

Now we consider the joint power and rate control problem with receiver faults at the master node.

Consider the following receiver outage fault model

$$
y^{F}(k)=(1-\rho) y(k)
$$

where $\rho=\operatorname{diag}\left[\rho_{1}, \rho_{2}, \cdots, \rho_{p}\right]$ and $\rho_{i}$ is an unknown constant with $\rho_{i}=1$ or $\rho_{i}=0$. When $\rho_{i}=1$, the receiver for the $i$ th node is outage; when $\rho_{i}=0$, there is no failure for the $i$ th node. The set of all possible $\rho$ is defined as $N_{\rho}$.

Then the joint power and rate control with receiver faults can be expressed as

$$
\begin{aligned}
& x(k+1)=A x(k)+B u(k)+B_{1} w(k), \\
& z(k)=C_{1} x(k), \\
& y(k)=(I-\rho) C x(k)+D w(k) .
\end{aligned}
$$

\section{E. Problem Formulation}

Definition 1: Consider the following system

$$
\begin{gathered}
x(k+1)=A_{a}(\hat{\rho}(k), \rho) x(k)+B_{a}(\hat{\rho}(k), \rho) w(k), \\
z(k)=C_{a}(\hat{\rho}(k), \rho) x(k),
\end{gathered}
$$

where $\rho$ is a parameter vector, and $\hat{\rho}(k)$ is a time-varying parameter vector to be chosen. Let $v>0$ be a given constant, then the system (21) is said to be with an adaptive $H_{\infty}$ performance index no larger than $v$, if for any $\varepsilon>0$, there exists a $\hat{\rho}(k)$ such that the following inequality holds

$$
\sum_{k=0}^{\infty} z^{T}(k) z(k) \leq v^{2} \sum_{k=0}^{\infty} w^{T}(k) w(k)+\varepsilon .
$$

Remark 3: From the above definition, for any $\eta>0$, let $\varepsilon=\eta^{2}$, then there exists a $\hat{\rho}(k)$ such that (23) holds. Thus, for $\sum_{k=0}^{\infty} w^{T}(k) w(k)>\eta$, we have

$$
\sum_{k=0}^{\infty} z^{T}(k) z(k) \leq\left(v^{2}+\eta\right) \sum_{k=0}^{\infty} w^{T}(k) w(k)
$$

for $\sum_{k=0}^{\infty} w^{T}(k) w(k) \leq \eta$, we have

$$
\sum_{k=0}^{\infty} z^{T}(k) z(k) \leq v^{2} \eta+\eta^{2}
$$

which shows that the adaptive $H_{\infty}$ performance index is close to the standard $H_{\infty}$ performance index when $\eta$ is sufficiently small.

The design problem under consideration is to find an adaptive controller for wireless networks with receiver faults described by (21) such that in both normal operation and fault cases, the resulting closed-loop system is asymptotically stable and its adaptive $H_{\infty}$ performance bound is minimized.

\section{ADAPTIVE FAULT-TOLERANT POWER AND RATE CONTROL}

Consider the joint power and rate control for wireless networks with receiver faults described by (21), the dynamic output feedback controller is chosen as

$$
\begin{gathered}
\xi(k+1)=A_{K}(\hat{\rho}) \xi(k)+B_{K}(\hat{\rho}) y^{F}(k), \\
u^{F}(k)=C_{K}(\hat{\rho}) \xi(k),
\end{gathered}
$$

where $\hat{\rho}(k)$ is the estimate of $\rho$,

$$
\begin{gathered}
\hat{\rho}(k)=\operatorname{diag}\left[\hat{\rho}_{1}(k) \cdots \hat{\rho}_{p}(k)\right], \\
A_{K}(\hat{\rho})=A_{K 0}+A_{K a}(\hat{\rho})+A_{K b}(\hat{\rho}), \\
B_{K}(\hat{\rho})=B_{K 0}+B_{K a}(\hat{\rho})+B_{K b}(\hat{\rho}),
\end{gathered}
$$

$$
A_{K a}(\hat{\rho})=\sum_{i=1}^{p} \hat{\rho}_{i} A_{K a i}
$$

$$
\begin{gathered}
A_{K b}(\hat{\rho})=\sum_{i=1}^{p} \hat{\rho}_{i} A_{K a i}+\sum_{i=1}^{p} \sum_{j=1}^{p} \hat{\rho}_{i} \hat{\rho}_{j} A_{K b i j}, \\
B_{K a}(\hat{\rho})=\sum_{i=1}^{p} \hat{\rho}_{i} B_{K a i}, \\
B_{K b}(\hat{\rho})=\sum_{i=1}^{p} \hat{\rho}_{i} B_{K b i}
\end{gathered}
$$

where $A_{K 0}, A_{K a i}, A_{K b i}, B_{K 0}, B_{K a i}, B_{K b i},(i, j=1, \cdots, p)$ and $C_{K}$ are fixed parameters to be designed.

From (21) and (26), we can obtain the following closed-loop system

$$
\begin{gathered}
x_{e}(k+1)=A_{e}(\hat{\rho}(k), \rho) x_{e}(k)+B_{e}(\hat{\rho}(k), \rho) w(k), \\
z_{e}(k)=C_{e} x_{e}(k),
\end{gathered}
$$

where $x_{e}(k)=\left[\begin{array}{lll}x^{T}(k) & \xi^{T}(k)\end{array}\right]^{T}$, and

$$
\begin{gathered}
A_{e}(\hat{\rho}(k), \rho)=\left[\begin{array}{cc}
A & B C_{K}(\hat{\rho}) \\
B_{K}(\hat{\rho})(I-\rho) C & A_{K}(\hat{\rho})
\end{array}\right], \\
B_{e}(\hat{\rho}(k), \rho)=\left[\begin{array}{c}
B_{1} \\
B_{K}(\hat{\rho})(I-\rho) D
\end{array}\right], \\
C_{e}=\left[\begin{array}{ll}
C_{1} & C_{K}
\end{array}\right] .
\end{gathered}
$$

The following notations will be used to present the main results. 


$$
\begin{aligned}
& N_{0 a}=\left[\begin{array}{ccc}
T_{0} & T_{1} & T_{2} \\
* & T_{3} & T_{4} \\
* & * & -v_{n}^{2} I
\end{array}\right], \\
& N_{0 b}=\left[\begin{array}{ccc}
T_{0} & T_{1} & T_{2} \\
* & T_{3} & T_{4} \\
* & * & -v_{f}^{2} I
\end{array}\right], \\
& R=\left[\begin{array}{llll}
R_{1} & R_{2} & \cdots & R_{p}
\end{array}\right], \\
& Q=\left[Q_{i j}\right] i, j=1,2, \cdots, p, \\
& R_{i}=\left[\begin{array}{ccc}
T_{5} & -N A_{K b i}-N_{3}^{T} N A_{K a i} & T_{6} \\
T_{7} & N A_{K b i} & T_{8} \\
0 & 0 & 0
\end{array}\right], \\
& Q_{i j}=\left[\begin{array}{ccc}
0 & T_{9} & 0 \\
T_{10} & N A_{K b i j}+\left(N A_{K b j i}\right)^{T} & T_{11} \\
0 & T_{12} & 0
\end{array}\right],
\end{aligned}
$$

With

$$
\begin{gathered}
T_{0}=Y A-N B_{K 0}(I-\rho) C \\
+\left(Y A-N B_{K 0}(I-\rho) C\right)^{T}+C_{1}^{T} C_{1} \\
T_{1}=Y B C_{K}-N A_{K 0}-N A_{K a}(\rho)-A^{T} N \\
+C^{T}(I-\rho) B_{K 0}^{T} N+C^{T} B_{K a}^{T}(\rho) N \\
+N_{3}^{T} N A_{K a}(\rho)-N_{3}^{T} C^{T} B_{K a}^{T}(\rho) N \\
T_{2}=Y B_{1}-N B_{K 0}(I-\rho) D \\
T_{3}=-N B C_{K}-\left(N B C_{K}\right)^{T}+N A_{K 0} \\
+N A_{K a}(\rho)+\left(N A_{K 0}+N A_{K a}(\rho)\right)^{T} \\
T_{4}=-N B_{1}+N B_{K 0}(I-\rho) D-A_{K a}^{T}(\rho) N N_{2} \\
+N B_{K a}(\rho) C N_{2} \\
+\left(N B_{K a i}-N B_{K b i} \rho\right) C N_{3} \\
T_{5}=\left(-N B_{K b i}-N B_{K a i}+N B_{K b i} \rho+N B_{K a i} \rho\right) C \\
T_{6}=-\left(N B_{K b i}+N B_{K a i}\right)(I-\rho) D \\
T_{7}=\left(-N B_{K a i} \rho+N B_{K b i}\right) C
\end{gathered}
$$

$$
\begin{gathered}
T_{8}=\left(N B_{K a i}+N B_{K b i}\right)(I-\rho) D+A_{K a i}^{T} N N_{2} \\
-\left(N B_{K a i}-N B_{K b i} \rho\right) C N_{2}, \\
T_{9}=-C^{T} B_{K b j}^{T} N-N A_{K b i j}+N_{3}^{T} C B_{K b j}^{T} N, \\
T_{10}=-N B_{K b i} C-A_{K b j i}^{T} N+N B_{K b i} C N_{3}, \\
T_{11}=N B_{K b i} C N_{2}, \\
T_{12}=-N_{2}^{T} C^{T} B_{K b j}^{T} N, \\
N_{1}=T_{c n}\left[\begin{array}{c}
T_{c} \\
0
\end{array}\right], \\
N_{2}=T_{c n}\left[\begin{array}{c}
T_{c}(I-\rho) D \\
0
\end{array}\right], \\
N_{3}=T_{c n}\left[\begin{array}{c}
T_{c} \rho C \\
C_{c n}
\end{array}\right],
\end{gathered}
$$

where $T_{c}$ is a matrix such that rank $\left(T_{C} C\right)=p_{1}<p$ and $C_{c n}$ is a matrix such that rank $\left[\begin{array}{l}T_{c} C \\ C_{c n}\end{array}\right]=n, T_{c n}=\left[\begin{array}{l}T_{c} C \\ C_{c n}\end{array}\right]^{-1}$.

The $\hat{\rho}_{i}(k)$ is determined by the following adaptive law

$$
\hat{\rho}_{i}(k+1)=\hat{\rho}_{i}(k)+\left\{\begin{array}{l}
0, \quad \hat{\rho}_{i}(k)=0 \text { and } L_{i} \leq 0 \\
L_{i}, \text { otherwise }
\end{array}\right.
$$

where

$$
\begin{gathered}
L_{i}=-l_{i}\left\{\xi ^ { T } ( k ) \left(N A_{K a i} \xi(k)-y^{F^{T}} N_{1}^{T} N A_{K a i} \xi(k)+\xi^{T}(k)\right.\right. \\
+\left[N B_{K a i} C+N B_{K b}(\hat{\rho}) C\right] N_{1} y^{F}
\end{gathered}
$$

and $l_{i} \geq 0(i=1,2, \cdots, m)$ is the adaptive law gain to be chosen according to the practical applications.

Theorem 1: Let $v_{f}>v_{n}>0$ be given constants. If there exist matrices $0<N<Y, A_{K 0}, A_{K a i}, A_{K b i}, B_{K 0}, B_{K a i}$, $B_{K b i}, C_{K}(i, j=1, \cdots, p)$ and a symmetric matrix $\Theta$ with

$$
\Theta=\left[\begin{array}{ll}
\Theta_{11} & \Theta_{12} \\
\Theta_{12}^{T} & \Theta_{22}
\end{array}\right],
$$

and $\Theta_{11}, \Theta_{22} \in R^{p(2 n+m) \times p(2 n+m)}$ such that the following LMIs hold

$$
\Theta_{22}^{i i} \leq 0, \quad i=1,2, \cdots, p,
$$

and for $\hat{\rho} \in \Delta_{\hat{\rho}}$ 


$$
\Theta_{11}+\Delta(\hat{\rho}) \Theta_{12}+\left[\Delta(\hat{\rho}) \Theta_{12}\right]^{T}+\Delta(\hat{\rho}) \Theta_{22} \Delta(\hat{\rho}) \geq 0
$$

for $\rho=0$ (i.e. in the normal case)

$$
\left[\begin{array}{cc}
N_{0 a} & R \\
R^{T} & Q
\end{array}\right]+G^{T} \Theta G<0
$$

and for $\rho \in N_{\rho}$ (i.e. in the failure cases)

$$
\left[\begin{array}{cc}
N_{0 b} & R \\
R^{T} & Q
\end{array}\right]+G^{T} \Theta G<0
$$

then the closed-loop system (27) via the dynamic output feedback controller (26) and the adaptive law (28) is asymptotically stable and with adaptive $H_{\infty}$ performance indexes no larger than $v_{n}$ and $v_{f}$ for normal and receiver failure cases, respectively. i.e., in the normal case $(\rho=0)$, satisfies

$$
\sum_{k=0}^{\infty} z^{T}(k) z(k) \leq v_{n}^{2} \sum_{k=0}^{\infty} w^{T}(k) w(k)+\sum_{i=1}^{p} \frac{\tilde{\rho}_{i}^{2}(0)}{l_{i}},
$$

and in receiver failure cases $(\rho \in N \rho)$, satisfies

$$
\sum_{k=0}^{\infty} z^{T}(k) z(k) \leq v_{f}^{2} \sum_{k=0}^{\infty} w^{T}(k) w(k)+\sum_{i=1}^{p} \frac{\tilde{\rho}_{i}^{2}(0)}{l_{i}},
$$

where $\tilde{\rho}(k)=\operatorname{diag}\left[\tilde{\rho}_{1}(k), \cdots, \tilde{\rho}_{p}(k)\right], \tilde{\rho}(k)=\hat{\rho}(k)-\rho$.

Proof: It is omitted here due to the length limitation.

\section{SiMULATION RESULTS}

To illustrate the performance of the proposed algorithm, we simulate a network using the model proposed in [2] for the channel gain from the $i$-th node to its master node. We take parameters according to the network [2]. $n(k)$ and $d(k)$ are zero-mean with variance 0.01 . Moreover, take $\bar{\mu}=0.8$ and $\alpha=0.2$.

Consider the following two possible fault cases: at $k=0$ and $k=10$, the receiver for node 1 is loss of $30 \%$ effectiveness and for other nodes are outage; at $k=5$, the receiver for node 2 is loss of $30 \%$ effectiveness and for the others are outage.

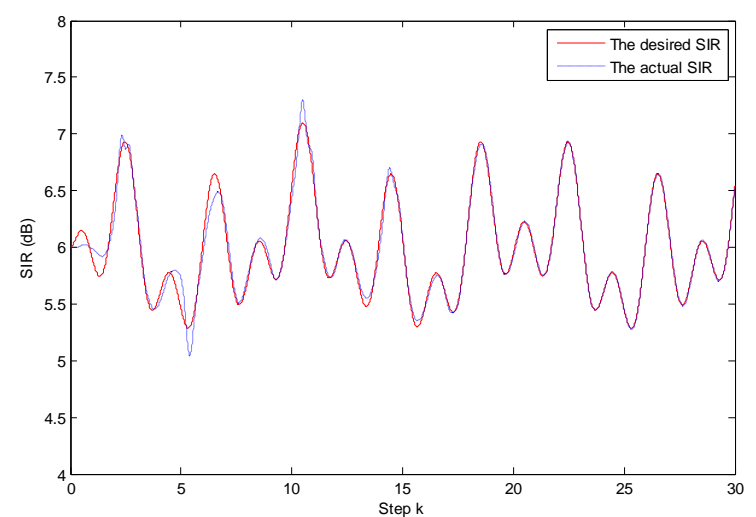

Fig. 1. Response curves of desired SIR and actual SIR in faulty cases with the proposed adaptive controller.
Fig. 1 and Fig. 2 describes the response curves of desired SIR and actual SIR in the above fault cases with the proposed adaptive fault-tolerant $H_{\infty}$ power and rate controller and fault-tolerant $H_{\infty}$ power and rate controller with fixed gains, respectively. It is easy to observe that the adaptive controller proposed in this paper has better performance than the power and rate controller with fixed gains in faulty cases.

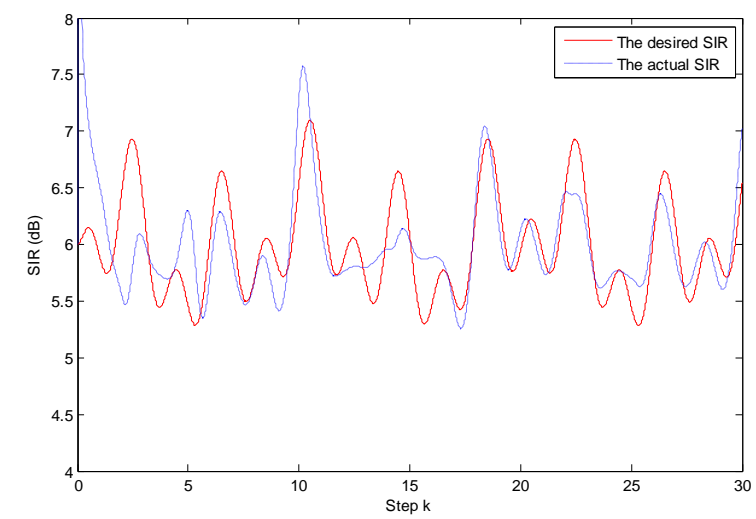

Fig. 2. Response curves of desired SIR and actual SIR in faulty cases with fault-tolerant $\mathrm{H} \infty$ controller with fixed gains.

\section{CONCLUSION}

This paper presents an adaptive fault-tolerant $H_{\infty}$ power and rate controller via dynamic output feedback for wireless networks with receiver faults. Based on the on-line estimation of eventual faults, the adaptive fault-tolerant controller parameters are updated automatically to compensate for the fault effect on the network. The adaptive $H_{\infty}$ performance is analyzed based on the linear matrix inequalities (LMIs). Simulation results show that the proposed adaptive controller can effectively compensate for the receiver faults and have better performance than fault-tolerant $H_{\infty}$ power and rate controller with fixed gains.

\section{REFERENCES}

[1] A. Subramanian and A. H. Sayed, "Joint rate and power control algorithms for wireless networks," IEEE Transactions on Signal Processing, vol. 53, no. 11, pp. 4204-4214, 2005.

[2] A. Subramanian and A. H. Sayed, "A robust power and rate control method for state-delayed wireless networks," Automatica, vol. 41, pp. 1917-1924, 2005

[3] S. L. Kong, H. S. Zhang, Z. S. Zhang, and C. H. Zhang, "Joint predictive control of power and rate for wireless networks," Acta Automatica Sinica, vol. 33, no. 7, pp.761-764, 2007.

[4] Y. L. Chen, Y. S. Lin, J. W. Wang, and J. H. Wen, "Adaptive fuzzy-based rate management and power control in multimedia CDMA cellular systems," Computer Communications, vol. 31, pp. 1901-1910, 2008.

[5] C. Fischione, M. Butussi, K. H. Johansson, and M. D. Angelo, "Power and rate control with outage constraints in CDMA wireless networks," IEEE Transactions on Communications, vol. 57, no. 8, pp. 2225-2229, 2009.

[6] T. Shu and M. Krunz, "Energy-efficient power/rate control and scheduling in hybrid TDMA/CDMA wireless sensor networks," Computer Networks, vol. 53, pp.1395-1408, 2009.

[7] A. Ghasemi and K. Faez, "Jointly rate and power control in contention based multihop wireless networks," Computer Communications, vol. 30, pp. 2021-2031, 2007.

[8] Z. Yang, S. Liao, and W. Cheng, "Joint power control and rate adaptation in wireless sensor networks," Ad Hoc Networks, vol. 7, pp. 401-410, 2009 
[9] K. Ramachandran, R. Kokku, H. Zhang, and M. Gruteser, "Symphony: synchronous two-phase rate and power control in 802.11 WLANs," IEEE/ACM Transactions on Networkings Communications, vol. 18, no. 4, pp. 1289-1302, 2010.

[10] C. Han, D. Sun, Z. Dong, Z. Li, and M. Zhao, "Adaptive fault-toleran $H \infty$ power and rate control for wireless networks via state feedback," Proceeding of the the $8^{\text {th }}$ Asian Control Conference, pp. 137-142, 2011.

[11] C. Han, D. Sun, and Z. Li, “Adaptive fault-tolerant $H \infty$ power and rate control for wireless networks via dynamic output feedback," Proceeding of World Congress on Intelligent Control and Automation, pp. 141-146, 2011.

[12] R. J. Veillette, J. V. Medanic, and W. R. Perkins, "Design of reliable control systems," IEEE Transactions on Automatic Control, vol. 37, no. 3, pp. 290-304, 1992.

[13] Q. Zhao and J. Jiang, "Reliable state feedback control system design against actuator failures," Automatica, vol. 34, no. 10, pp. 1267-1272, 1998.

[14] F. Liao, J. L. Wang, and G. H. Yang, "Reliable robust flignt tracking control: an LMI approach," IEEE Transactions on Control Systems Technology, vol. 10, no. 1, pp. 76-89, 2002.

[15] J. Stoustrup and V. D. Blondel, "Fault tolerant control: a simultaneous stabilization result," IEEE Transactions on Automatic Control, vol. 49, no. 2, pp. 305-310, 2004.

[16] H. N. Wu and H. Y. Zhang, "Reliable $\mathrm{H} \infty$ fuzzy control for continuous-time nonlinear systems with actuator failures," IEEE Transactions on fuzzy systems, vol. 14, no. 5, pp. 609-618, 2006.
[17] G. Pujol, J. Rodellar, J. M. Rossell, and F. Pozo, "Decentralised reliable guaranteed cost control of uncertain systems: An LMI design," Proc.Inst. Elect. Eng., vol. 1, no. 3, pp. 779-785, 2007

[18] G. Tao, S. M. Joshi, and X. L. Ma, "Adaptive state feedback and tracking control of systems with actuator failures," IEEE Transations on Automatic Control, vol. 46, no. 1, pp. 78-95, 2001.

[19] X. D. Tang, G. Tao, and L. F. Wang, "Robust and adaptive actuator failure compensation designs for a rocket fairing structural-acoustic model," IEEE Transactions on Aerospace and Electronic Systems, vol. 40, no. 4, pp. 1359-1366, 2004.

[20] D. Ye and G. H. Yang, "Adaptive fault-tolerant tracking control against actuator faults with application to flight control," IEEE Transactions on Control Systems Technology, vol. 14, no. 6, pp. 1088-1096, 2006.

[21] G. H. Yang and D. Ye, "Adaptive reliable Hळ filtering against sensor failures," IEEE Transactions on Signal Processing, vol. 55, no. 7, pp. 3161-3171, 2007

[22] G. H. Yang and D. Ye, "Adaptive fault-tolerant $\mathrm{H} \infty$ control against sensor faults," IET Control Theory and Applicationsl, vol. 2, no. 2, pp. 95-107, 2008.

[23] G. H. Yang and D. Ye, "Reliable Hœ control of linear systems with adaptive mechanism," IEEE Transactions on Automatic Control, vol. 55, no. 1, pp. 242-247, 2010.

[24] F. P. Kelly, "Models for a self managed internet," Philosophical Transactions of the Royal Society, vol. 358, pp. 2335-2348, 2000. 